\title{
TAXONOMIA E DISTRIBUIÇÃO DE ACTINOCYTHEREIS BRASILIENSIS SP. NOV. (PODOCOPIDA, TRACHYLEBERIDIDAE) NA PLATAFORMA CONTINENTAL BRASILEIRA
}

\author{
Cláudia P. Machado ${ }^{1}$ \\ Núscia G. S. Drozinski ${ }^{2}$
}

\begin{abstract}
TAXONOMY AND DISTRIBUTION OF ACTINOCYTHEREIS BRASILIENSIS SP. NOV. (PODOCOPIDA, TRACHYLEBERIDIDAE) FROM THE BRAZILIAN CONTINENTAL SHELF. A new species is described, Actinocythereis brasiliensis, from the southern Brazilian continental shelf. It is based on the analysis of 766 samples collected by oceanographical projects. The geographical distribution of this species has revealed its preference by the cold waters and terrigenous sediments and, has a bathymetric distribution between 22 and $164 \mathrm{~m}$ but, being concentrated in the shallower waters $(<60 \mathrm{~m})$.
\end{abstract}

KEYWORDS. Brazilian Continental Shelf, Ostracoda, Crustacea, Taxonomy, Zoogeography.

\section{INTRODUÇÃO}

O gênero Actinocythereis foi proposto por PURI (1953), tendo como espécie-tipo Cythere exanthemata Ulrich \& Bassler, 1904; foram incluídas oito espécies no gênero, todas fósseis (Eoceno-Mioceno). Moore \& Pitrat (1961), Mckenzie (1967) e Hazel (1967) aceitaram a proposta do novo gênero e estenderam sua ocorrência para outros períodos da era Cenozóica, inclusive o Recente. Entretanto, alguns autores como MoRKHOVEn (1963), consideraram Actinocythereis subgênero de Trachyleberis Brady, 1898, devido a relativa similaridade entre a ornamentação das suas espécies. Contudo, é possível separar Actinocythereis de Trachyleberis por suas características morfológicas, inclusive a ornamentação: o primeiro apresenta três fileiras longitudinais de projeções espiniformes e/ou nodulosas, algumas vezes intercaladas por pequenos a relativamente amplos espaços entre os espinhos e/ou nódulos, e Trachyleberis possui ornamentação muito mais dispersa e irregular.

\footnotetext{
1. Programa de Pós-graduação em Biologia Animal, Universidade Federal do Rio Grande do Sul, Av. Bento Gonçalves, 9500 , P 43435, sala 101, 91501-970 Porto Alegre, RS, Brasil. (claudinhacpm@yahoo.com.br)

2. Programa de Pós-graduação em Geociências (Paleontologia), Universidade Federal do Rio Grande do Sul, Caixa Postal 15001, 91501-970, Porto Alegre, RS, Brasil.
} 
Estudos deste gênero no Brasil têm se mostrado escassos, sendo seu primeiro registro na plataforma continental brasileira realizado por PINTO et al. (1978). Objetivase descrever uma nova espécie e delimitar suas distribuições geográfica e batimétrica na costa brasileira.

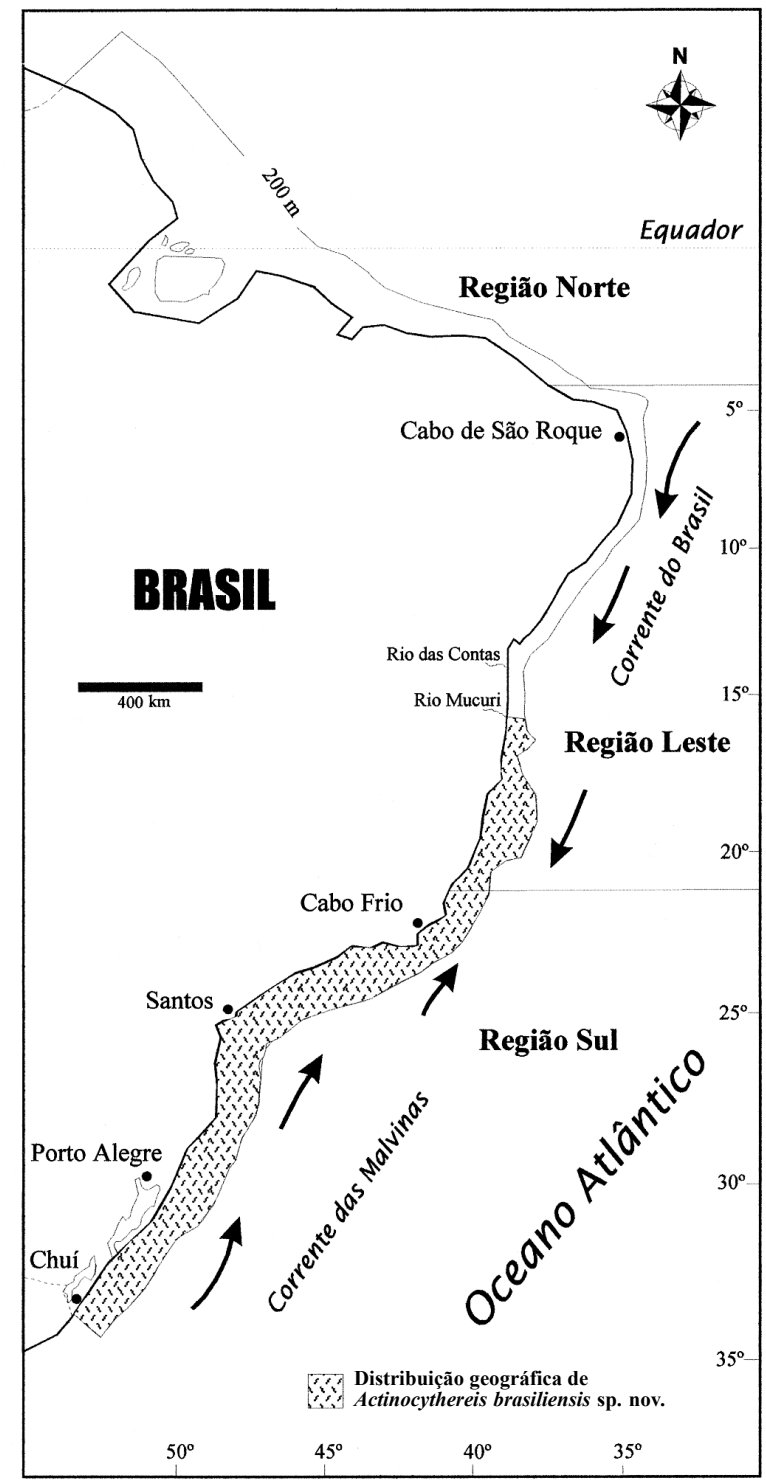

Fig. 1. Distribuição geográfica de Actinocythereis brasiliensis sp. nov. na plataforma continental brasileira. Modificado de CHAVES (1983).

\section{MATERIAL E MÉTODOS}

A área de estudo compreende toda a extensão da plataforma continental brasileira, localizada entre as latitudes $4^{\circ} 25^{\prime} \mathrm{N}$ e 3345'S. Chaves (1983) a dividiu em três regiões, norte, leste e sul, de acordo com suas características geológicas e geomorfológicas (fig. 1). A região norte apresenta predomínio de sedimentos terrígenos sobre os carbonáticos, estando estes últimos restritos à plataforma externa e em pontos isolados da plataforma interna (Coimbra et al., 1995). A sedimentação na plataforma leste é caracterizada basicamente por areias biodetríticas ortoquartizíticas e construções carbonáticas, presentes entre o Cabo de São Roque e Rio das Contas, e sedimentação terrígena desde este último até a cidade de Cabo Frio (Francisconi et al., 1974). No entanto, as fácies terrígenas tornamse significativas somente ao longo da costa do estado do Ceará e na região das desembocaduras de grandes rios, como o São Francisco, Mucuri, São Mateus e Doce (Kowsmann \& Costa, 1979). A plataforma leste possui menor largura do que as demais e sua profundidade máxima está em torno de $60 \mathrm{~m}$ (KempF, 1970).

A plataforma sul apresenta dois domínios sedimentares distintos, um formado predominantemente por sedimentos terrígenos nas plataformas interna e média, e outro constituído por áreas carbonáticas na plataforma externa (Kowsmann \& Costa, 1979). Segundo aqueles autores, as fácies sedimentares terrígenas apresentam-se bastante homogêneas ao longo de toda a região sul e as fácies carbonáticas aparecem 
de Cabo Frio a Santos, surgindo em pontos isolados na plataforma interna. A profundidade da plataforma continental nesta região pode chegar a $160 \mathrm{~m}$.

Duas grandes correntes possuem forte influência nas regiões leste e sul, a Corrente das Malvinas ou Falklands, com temperaturas que variam de $4^{\circ} \mathrm{C}$, próximo às Ilhas Malvinas, a $20^{\circ} \mathrm{C}$, próximo à latitude $32^{\circ} \mathrm{S}$, e a Corrente do Brasil com temperaturas em torno de $26^{\circ} \mathrm{C}$ (MARTins, 1984). A Corrente das Malvinas tem sua origem em águas subantárticas, e a do Brasil surge como uma subdivisão da Corrente Equatorial, que se desloca em direção sul (fig. 1). A região de Cabo Frio $\left(23^{\circ} \mathrm{S}\right)$ é uma zona de ressurgência, constituindo-se no último remanescente da Corrente das Malvinas na costa brasileira (Coimbra \& Ornellas, 1989; Stevenson et al., 1998).

Foram analisadas 766 amostras provenientes da plataforma continental brasileira, obtidas através do Projeto REMAC (pernadas I, II, III e VII), Operações GEOMAR III e VI, Navio Oceanográfico "El Austral”, Projeto GEOCOSTA RIO-II e Programa REVIZEE - Score Sul. As coletas foram realizadas com auxílio de amostrador Van Veen e Box corer, em profundidades de até $500 \mathrm{~m}$ e o processamento das amostras, realizado através de técnicas de laboratório usuais para a preparação e o estudo de carapaças de ostracodes marinhos. As fotomicrografias foram tomadas em câmera fotográfica, acoplada em microscópio óptico para as imagens por transparência, e em microscópio eletrônico de varredura (MEV), Centro de Microscopia Eletrônica, Universidade Federal do Rio Grande do Sul. As valvas das fêmeas, utilizadas para fotografias em luz transmitida (figs. 11-14), foram posteriormente danificadas, impossibilitando o depósito na coleção do Museu de Paleontologia. As seguintes abreviaturas foram utilizadas na descrição dos espécimens: $\mathrm{C}$, comprimento; $\mathrm{L}$, largura; $\mathrm{h}$, altura; $\mathrm{Ca}$, carapaça; $\mathrm{VD}$, valva direita e VE, valva esquerda.

O material-tipo está depositado na coleção do Museu de Paleontologia, Departamento de Paleontologia e Estratigrafia, Universidade Federal do Rio Grande do Sul, Seção de Ostracoda (MP-O).

\section{Actinocythereis brasiliensis sp. nov.}

(Figs. 2-14)

\section{Etimologia. O nome específico é alusivo à plataforma continental brasileira.}

Material-tipo. Holótipo $\odot$, MP-O 1745, VD, C=0,6806 mm, h=0,3984 mm; parátipos: $\odot$, MP-O 1746, VE, C=0,6972 mm, h=0,4150 mm; , MP-O 1747, VE, C=0,6972 mm, h=0,4150 mm; ठ, MP-O 1748, VD, C=0,7138 mm, h=0,3735 mm; o', MP-O 1749, VE, C=0,6972 mm, h=0,3818 mm; todos do Programa REVIZEE Score-Sul, amostra 6851, $31^{\circ} 57^{\prime}$ 'S, 5009' W, 06.IV.1998. Projeto GEOCOSTA RIO II:

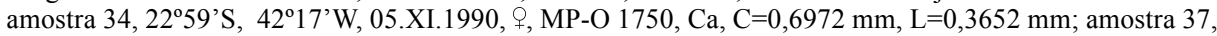

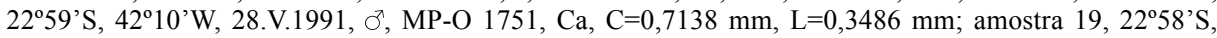
$42^{\circ} 05^{\prime}$ W, 10.IX.1990, Ј', MP-O 1752, VE, C $=0,7138 \mathrm{~mm}, \mathrm{~h}=0,3818 \mathrm{~mm}$.

Diagnose. Carapaça pequena, lateralmente sub-retangular a subtrapezoidal. Superfície ornamentada por tubérculos de diferentes tamanhos, freqüentemente unidos, dispostos em três fileiras longitudinais; a mediana, mais espessa no extremo anterior, com uma descontinuidade em relação ao tubérculo subcentral, abaixo da qual encontrase um bem-desenvolvido tubérculo isolado. Tubérculo subcentral irregular anastomosado a outros tubérculos. Porocanais normais, abrem-se no topo dos tubérculos.

Diagnosis. A small species of Actinocythereis, subrectangular to subtrapezoidal in lateral view. Surface with three longitudinal ribs constituted by tubercles of different sizes, frequently anastomosed. A well developed tubercle bellow the discontinuity between the subcentral tubercle and the median rib. Subcentral tubercle very irregular. Normal pore canals opened in the top of the tubercles.

Descrição (figs. 2-14). Carapaça pequena, sub-retangular a subtrapezoidal em vista lateral; extremos anterior e posterior lateralmente comprimidos; VE maior que VD. Maior altura junto ao ângulo cardinal anterior, no qual situa-se um tubérculo ocular proeminente e arredondado. Margens dorsal e ventral sub-retilíneas, suavemente convergentes em direção à região posterior. Margem anterior ampla e obliquamente arredondada, sustentando duas fileiras de espinhos. Margem posterior medianamente 


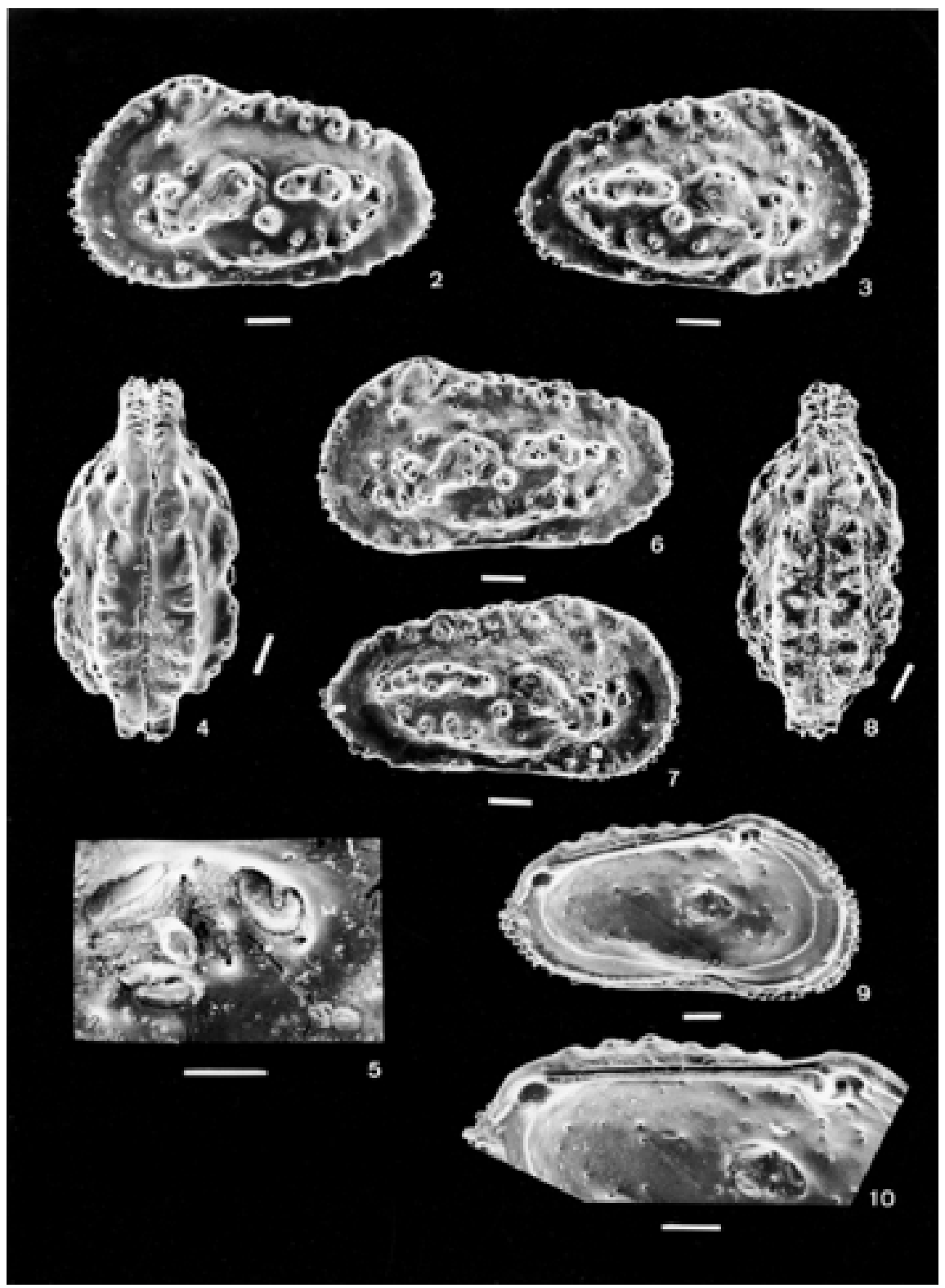

Figs. 2-10. Actinocythereis brasiliensis sp. nov.: 2, parátipo $९$, VE (MP-O 1746); 3, holótipo 9 , VD (MP-O 1745); 4, parátipo $9, \mathrm{Ca}$, vista dorsal (MP-O 1750); 5, parátipo $\odot$, VE, vista interna, detalhe da impressão muscular (MP-O 1747); 6, parátipo $\sigma^{7}$, VE (MP-O 1749); 7, parátipo $\sigma^{7}$, VD (MP-O-1748); 8, parátipo $\sigma^{7}$, Ca, vista dorsal (MP-O 1751); 9, parátipo ơ, VE, vista interna (MP-O 1752); 10, parátipo ơ , detalhe da charneira (MP-O 1752). Barras: figs. 2-4, 6-10, $100 \mu \mathrm{m}$; fig. 5, $50 \mu \mathrm{m}$. 
Tabela I. Relação das amostras (A) por projeto, coordenadas, profundidade (m) e sedimentologia (S), que acusaram a presença de Actinocythereis brasiliensis sp. nov. Pernadas do REMAC, RP; Navio El Austral, AUS; GEOMAR VII, GM; GEOCOSTA RIO II, GC; REVIZEE, RZ. Sedimentologia: lama, L; lama arenosa, La; lama biodetrítica, Lb; lama arenosa biodetrítica, Lab; silte, S; silte biodetrítico, Sb; silte lamonoso, Sl; areia, A; areia fina, Af; areia fina biodetrítica, $\mathrm{Afb}$; areia biodetrítica, $\mathrm{Ab}$; areia fina lamosa, Afl; areia lamosa, $\mathrm{Al}$; areia lamosa biodetrítica, Alb; areia média, Am; areia média lamosa, Aml; areia grossa biodetrítica, Agb.

\begin{tabular}{|c|c|c|c|c|c|c|c|c|c|}
\hline Projeto & A & Coordenadas & (m) & S & Projeto & A & Coordenadas & $(\mathrm{m})$ & $\mathrm{S}$ \\
\hline \multirow[t]{9}{*}{ RP I } & 3010 & $26^{\circ} 30^{\prime} \mathrm{S}, 48^{\circ} 15^{\prime} \mathrm{W}$ & 46 & $\mathrm{Lb}$ & RP VII & 3834 & $16^{\circ} 49^{\prime} \mathrm{S}, 39^{\circ} 00^{\prime} \mathrm{W}$ & 22 & $\mathrm{Ab}$ \\
\hline & 3011 & $26^{\circ} 44^{\prime} \mathrm{S}, 48^{\circ} 16^{\prime} \mathrm{W}$ & 49 & $\mathrm{Lb}$ & & 3915 & $19^{\circ} 53^{\prime} \mathrm{S}, 39^{\circ} 55^{\prime} \mathrm{W}$ & 37 & $\mathrm{Al}$ \\
\hline & 3012 & $26^{\circ} 55^{\prime} \mathrm{S}, 48^{\circ} 17^{\prime} \mathrm{W}$ & 47 & $\mathrm{Lb}$ & & 3923 & $20^{\circ} 36^{\prime} \mathrm{S}, 40^{\circ} 02^{\prime} \mathrm{W}$ & 55 & $\mathrm{Ab}$ \\
\hline & 3030 & $29^{\circ} 52^{\prime} \mathrm{S}, 49^{\circ} 45^{\prime} \mathrm{W}$ & 40 & $\mathrm{Lab}$ & & 3929 & $22^{\circ} 01^{\prime} \mathrm{S}, 40^{\circ} 35^{\prime} \mathrm{W}$ & 42 & $\mathrm{Ab}$ \\
\hline & 3031 & $30^{\circ} 01^{\prime} \mathrm{S}, 49^{\circ} 52^{\prime} \mathrm{W}$ & 38 & Lab & & 3955 & $22^{\circ} 52^{\prime} \mathrm{S}, 41^{\circ} 16^{\prime} \mathrm{W}$ & 70 & $\mathrm{Alb}$ \\
\hline & 3067 & $32^{\circ} 28^{\prime} \mathrm{S}, 51^{\circ} 01^{\prime} \mathrm{W}$ & 61 & $\mathrm{La}$ & & 3957 & $22^{\circ} 37^{\prime} \mathrm{S}, 41^{\circ} 39^{\prime} \mathrm{W}$ & 46 & $\mathrm{Alb}$ \\
\hline & 3086 & $33^{\circ} 51^{\prime} \mathrm{S}, 51^{\circ} 42^{\prime} \mathrm{W}$ & 87 & $\mathrm{Alb}$ & & 3961 & $22^{\circ} 42^{\prime} \mathrm{S}, 41^{\circ} 51^{\prime} \mathrm{W}$ & 37 & $\mathrm{Ab}$ \\
\hline & 3096 & $29^{\circ} 52^{\prime} \mathrm{S}, 49^{\circ} 42^{\prime} \mathrm{W}$ & 43 & Lab & & 3963 & $23^{\circ} 07^{\prime} \mathrm{S}, 42^{\circ} 55^{\prime} \mathrm{W}$ & 57 & $\mathrm{La}$ \\
\hline & 3105 & $31^{\circ} 42^{\prime} \mathrm{S}, 50^{\circ} 41^{\prime} \mathrm{W}$ & 78 & Lab & AUS & 6701 & $28^{\circ} 39^{\prime} \mathrm{S}, 48^{\circ} 41^{\prime} \mathrm{W}$ & 48 & $\mathrm{~L}$ \\
\hline \multirow[t]{10}{*}{ RP II } & 3163 & $26^{\circ} 56^{\prime} \mathrm{S}, 48^{\circ} 25^{\prime} \mathrm{W}$ & 36 & Lab & & 6702 & $28^{\circ} 51^{\prime} \mathrm{S}, 48^{\circ} 48^{\prime} \mathrm{W}$ & 46 & $\mathrm{~L}$ \\
\hline & 3171 & $25^{\circ} 24^{\prime} \mathrm{S}, 47^{\circ} 06^{\prime} \mathrm{W}$ & 58 & $\mathrm{Lab}$ & & 6703 & $28^{\circ} 56^{\prime} \mathrm{S}, 49^{\circ} 07^{\prime} \mathrm{W}$ & 38 & $\mathrm{La}$ \\
\hline & 3195 & $24^{\circ} 41^{\prime} \mathrm{S}, 45^{\circ} 02^{\prime} \mathrm{W}$ & 116 & $\mathrm{Ab}$ & & 6704 & $29^{\circ} 04^{\prime} \mathrm{S}, 49^{\circ} 15^{\prime} \mathrm{W}$ & 38 & $\mathrm{La}$ \\
\hline & 3203 & $24^{\circ} 20^{\prime} \mathrm{S}, 45^{\circ} 43^{\prime} \mathrm{W}$ & 60 & $\mathrm{Lab}$ & & 6714 & $31^{\circ} 38^{\prime} \mathrm{S}, 51^{\circ} 00^{\prime} \mathrm{W}$ & 37 & $\mathrm{Al}$ \\
\hline & 3204 & $24^{\circ} 30^{\prime} \mathrm{S}, 45^{\circ} 56^{\prime} \mathrm{W}$ & 58 & $\mathrm{Alb}$ & & 6738 & $35^{\circ} 06^{\prime} \mathrm{S}, 53^{\circ} 37^{\prime} \mathrm{W}$ & 37 & A \\
\hline & 3205 & $24^{\circ} 40^{\prime} \mathrm{S}, 46^{\circ} 18^{\prime} \mathrm{W}$ & 58 & Afb & GM & G 333 & $32^{\circ} 57^{\prime} \mathrm{S}, 50^{\circ} 28^{\prime} \mathrm{W}$ & 120 & $\mathrm{Lab}$ \\
\hline & 3206 & $24^{\circ} 49^{\prime} \mathrm{S}, 46^{\circ} 35^{\prime} \mathrm{W}$ & 52 & Afb & & G 346 & $31^{\circ} 51^{\prime} \mathrm{S}, 51^{\circ} 10^{\prime} \mathrm{W}$ & 44 & $\mathrm{Alb}$ \\
\hline & 3211 & $25^{\circ} 45^{\prime} \mathrm{S}, 47^{\circ} 12^{\prime} \mathrm{W}$ & 74 & $\mathrm{Alb}$ & & G 349 & $31^{\circ} 24^{\prime} \mathrm{S}, 50^{\circ} 41^{\prime} \mathrm{W}$ & 60 & $\mathrm{Alb}$ \\
\hline & 3215 & $26^{\circ} 14^{\prime} \mathrm{S}, 46^{\circ} 35^{\prime} \mathrm{W}$ & 164 & Afb & & G 350 & $31^{\circ} 29^{\prime} \mathrm{S}, 50^{\circ} 30^{\prime} \mathrm{W}$ & 96 & $\mathrm{Lab}$ \\
\hline & 3221 & $24^{\circ} 47^{\prime} \mathrm{S}, 46^{\circ} 10^{\prime} \mathrm{W}$ & 60 & Lab & & G 362 & $31^{\circ} 06^{\prime} \mathrm{S}, 49^{\circ} 46^{\prime} \mathrm{W}$ & 135 & $\mathrm{Ab}$ \\
\hline \multirow[t]{18}{*}{ RP III } & 3227 & $24^{\circ} 46^{\prime} \mathrm{S}, 46^{\circ} 40^{\prime} \mathrm{W}$ & 46 & $\mathrm{Afb}$ & & G 370 & $30^{\circ} 37^{\prime} \mathrm{S}, 49^{\circ} 06^{\prime} \mathrm{W}$ & 148 & $\mathrm{Alb}$ \\
\hline & 3228 & $25^{\circ} 03^{\prime} \mathrm{S}, 46^{\circ} 39^{\prime} \mathrm{W}$ & 51 & Afb & $\mathrm{GC}$ & 17 & $22^{\circ} 59^{\prime} \mathrm{S}, 42^{\circ} 03^{\prime} \mathrm{W}$ & 53 & Af \\
\hline & 3236 & $23^{\circ} 37^{\prime} \mathrm{S}, 44^{\circ} 37^{\prime} \mathrm{W}$ & 51 & $\mathrm{Al}$ & & 18 & $22^{\circ} 59^{\prime} \mathrm{S}, 42^{\circ} 04^{\prime} \mathrm{W}$ & 50 & $\mathrm{Am}$ \\
\hline & 3244 & $23^{\circ} 11^{\prime} \mathrm{S}, 43^{\circ} 32^{\prime} \mathrm{W}$ & 51 & $\mathrm{Ab}$ & & 19 & $22^{\circ} 58^{\prime} \mathrm{S}, 42^{\circ} 05^{\prime} \mathrm{W}$ & 42 & $\mathrm{Aml}$ \\
\hline & 3245 & $23^{\circ} 17^{\prime} \mathrm{S}, 43^{\circ} 47^{\prime} \mathrm{W}$ & 54 & $\mathrm{~L}$ & & 31 & $22^{\circ} 58^{\prime} \mathrm{S}, 42^{\circ} 18^{\prime} \mathrm{W}$ & 48 & $\mathrm{Aml}$ \\
\hline & 3246 & $23^{\circ} 24^{\prime} \mathrm{S}, 44^{\circ} 05^{\prime} \mathrm{W}$ & 57 & $\mathrm{Sb}$ & & 33 & $22^{\circ} 59^{\prime} \mathrm{S}, 42^{\circ} 19^{\prime} \mathrm{W}$ & 51 & Afl \\
\hline & 3247 & $23^{\circ} 30^{\prime} \mathrm{S}, 44^{\circ} 20^{\prime} \mathrm{W}$ & 55 & $\mathrm{~L}$ & & 34 & $22^{\circ} 59^{\prime} \mathrm{S}, 42^{\circ} 17^{\prime} \mathrm{W}$ & 49 & $\mathrm{Agb}$ \\
\hline & 3248 & $23^{\circ} 44^{\prime} \mathrm{S}, 44^{\circ} 24^{\prime} \mathrm{W}$ & 70 & S & & 36 & $22^{\circ} 59^{\prime} \mathrm{S}, 42^{\circ} 12^{\prime} \mathrm{W}$ & 50 & Afl \\
\hline & 3249 & $23^{\circ} 57^{\prime} \mathrm{S}, 44^{\circ} 27^{\prime} \mathrm{W}$ & 115 & $\mathrm{~L}$ & & 37 & $22^{\circ} 59^{\prime} \mathrm{S}, 42^{\circ} 10^{\prime} \mathrm{W}$ & 51 & Afl \\
\hline & 3252 & $23^{\circ} 42^{\prime} \mathrm{S}, 43^{\circ} 44^{\prime} \mathrm{W}$ & 110 & $\mathrm{~L}$ & & 39 & $22^{\circ} 59^{\prime} \mathrm{S}, 42^{\circ} 06^{\prime} \mathrm{W}$ & 58 & Afl \\
\hline & 3254 & $23^{\circ} 26^{\prime} \mathrm{S}, 42^{\circ} 14^{\prime} \mathrm{W}$ & 106 & $\mathrm{~L}$ & & 43 & $23^{\circ} 00^{\prime} \mathrm{S}, 42^{\circ} 05^{\prime} \mathrm{W}$ & 57 & Afb \\
\hline & 3257 & $23^{\circ} 01^{\prime} \mathrm{S}, 42^{\circ} 30^{\prime} \mathrm{W}$ & 64 & $\mathrm{Ab}$ & & 46 & $23^{\circ} 00^{\prime} \mathrm{S}, 42^{\circ} 11^{\prime} \mathrm{W}$ & 62 & Af \\
\hline & 3260 & $23^{\circ} 04^{\prime} \mathrm{S}, 41^{\circ} 20^{\prime} \mathrm{W}$ & 80 & $\mathrm{~L}$ & & 47 & $23^{\circ} 00^{\prime} \mathrm{S}, 42^{\circ} 13^{\prime} \mathrm{W}$ & 58 & Afl \\
\hline & 3264 & $23^{\circ} 31^{\prime} \mathrm{S}, 41^{\circ} 32^{\prime} \mathrm{W}$ & 128 & $\mathrm{Ab}$ & & 48 & $23^{\circ} 00^{\prime} \mathrm{S}, 42^{\circ} 16^{\prime} \mathrm{W}$ & 58 & Afl \\
\hline & 3280 & $23^{\circ} 23^{\prime} \mathrm{S}, 41^{\circ} 26^{\prime} \mathrm{W}$ & 115 & $\mathrm{Ab}$ & & 49 & $23^{\circ} 00^{\prime} \mathrm{S}, 42^{\circ} 18^{\prime} \mathrm{W}$ & 60 & Afl \\
\hline & 3287 & $22^{\circ} 33^{\prime} \mathrm{S}, 41^{\circ} 34^{\prime} \mathrm{W}$ & 46 & $\mathrm{Al}$ & & 51 & $23^{\circ} 01^{\prime} \mathrm{S}, 42^{\circ} 17^{\prime} \mathrm{W}$ & 67 & $\mathrm{Sl}$ \\
\hline & 3288 & $22^{\circ} 42^{\prime} \mathrm{S}, 41^{\circ} 49^{\prime} \mathrm{W}$ & 42 & $\mathrm{~S}$ & $\mathrm{RZ}$ & 6851 & $31^{\circ} 57^{\prime} \mathrm{S}, 50^{\circ} 09^{\prime} \mathrm{W}$ & 160 & $\mathrm{La}$ \\
\hline & 3312 & $19^{\circ} 30^{\prime} \mathrm{S}, 39^{\circ} 37^{\prime} \mathrm{W}$ & 29 & $\mathrm{Am}$ & & & & & \\
\hline
\end{tabular}

angulosa, com duas fileiras de espinhos na metade inferior. Superfície ornamentada por tubérculos de diferentes tamanhos, freqüentemente anastomosados, dispostos em três fileiras longitudinais; a central, mais espessa no extremo anterior, apresenta uma descontinuidade em relação ao tubérculo subcentral, abaixo da qual encontra-se um tubérculo bem-desenvolvido isolado. Porocanais normais, simples ou crivados, abremse no topo dos tubérculos. Duas amplas costelas marginais destacam-se nas regiões 


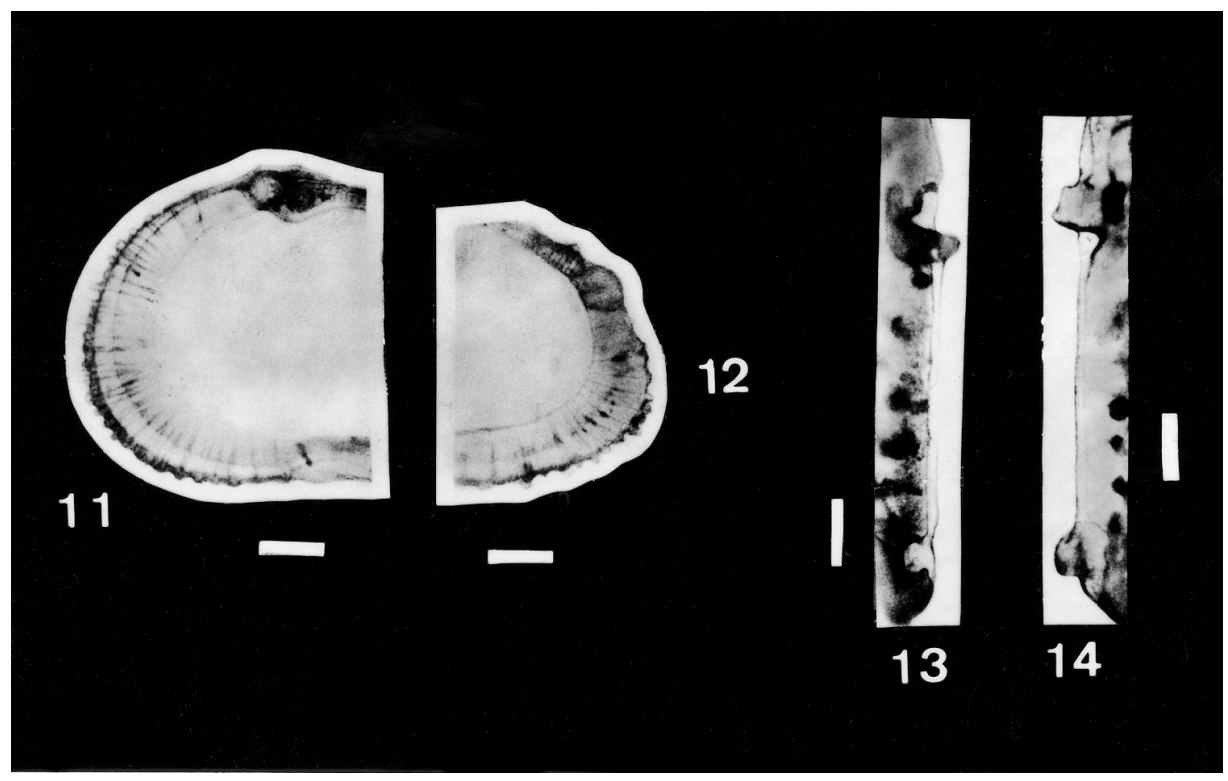

Figs. 11-14. Actinocythereis brasiliensis sp. nov.,, : 11, 12, vista lateral interna, detalhe dos porocanais marginais, VD: 11, anteriores; 12, posteriores. 13, detalhe da charneira, VE; 14, detalhe da charneira, VD. Barras: $100 \mu \mathrm{m}$.

anterior e posterior. Tubérculo subcentral anastomosado a outros tubérculos, assumindo configuração irregular. Em vista dorsal, carapaça medianamente comprimida; metades anterior e posterior com larguras coincidentes. Em vista interna, charneira holanfidonte; elemento póstero-mediano finamente crenulado. Duplicatura ampla, com porocanais marginais numerosos, simples, retilíneos, podendo formar pares e ocasionalmente apresentar bifurcações. Linha de concrescência e margem interna coincidentes. Impressões musculares localizadas em depressão subcentral, com a frontal em forma de "V" e uma fila vertical de quatro adutoras alongadas, a superior obliquamente disposta. Impressão mandibular imediatamente abaixo da frontal. Dimorfismo sexual nítido: machos mais compridos, baixos e estreitos.

Discussão. Actinocythereis brasiliensis é similar a A. exanthemata (Ulrich \& Bassler,1904), espécie-tipo do gênero. Difere pelo contorno subtrapezoidal, tamanho menor e costelas formadas por tubérculos em vez de espinhos.

Ocorrência. Das 766 amostras analisadas, apenas 73 registraram A. brasiliensis. A espécie é distribuída na plataforma continental sulbrasileira, apresentando ocorrência restrita à porção meridional da plataforma leste (fig.1, tab. I). O registro mais ao sul,

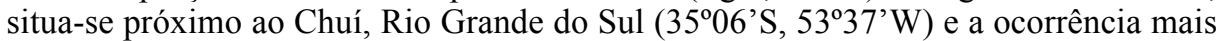
setentrional $\left(16^{\circ} 49^{\prime} \mathrm{S}, 3^{\circ} 00^{\prime} \mathrm{W}\right)$, próximo à desembocadura do rio Mucuri, Bahia. A distribuição batimétrica varia de $22 \mathrm{~m}$ (no extremo setentrional) até $164 \mathrm{~m}$ (na plataforma externa do norte de Santa Catarina). Actinocythereis brasiliensis é comum em profundidades inferiores a $60 \mathrm{~m}$ (tab. I). A zona de ressurgência marinha não marcou o limite da distribuição da espécie entre as regiões sul e leste, como sugeriram BoLTOVSKOY 
(1976) e ForTi-Esteves (1984) em estudos de foraminíferos e moluscos, respectivamente. Entretanto, a preferência de $A$. brasiliensis por sedimentos terrígenos e águas mais frias parece determinante na sua distribuição ao longo da costa. A distribuição zoogeográfica da espécie aqui estudada permitiu enquadrá-la dentro das assembléias sul e transicional, reconhecidas por CoIMBRA \& ORNELlas (1989), e apoiadas por CoIMBRA et al. (1995), Ramos (1996), Fauth \& Coimbra (1998) e CARmo \& SAnguinetTi (1999). A ausência desta espécie na porção mais setentrional da plataforma leste e em toda a norte, pode estar relacionada com a presença de sedimentos carbonáticos e águas mais quentes nestas áreas, que atuariam como barreiras para a dispersão deste microcrustáceo.

Distribuição. Recente, na plataforma continental sul-brasileira e na porção mais meridional da leste (fig. 1).

Agradecimentos. Ao Luís Flávio Lopes pelo auxílio nos trabalhos fotográficos, ao Cristianini Trescastro Bergue, pelas discussões e auxílio no MEV, e ao Prof. Dr. João Carlos Coimbra (UFRGS) que proporcionou o apoio indispensável para o desenvolvimento deste trabalho.

\section{REFERÊNCIAS BIBLIOGRÁFICAS}

Boltovsкoy, E. 1976. Distribution of recent Foraminifera of the South America Region. In: Hedley, R. H. \& Adams, C. G. eds. Foraminifera. London, Academic. v.2, p.171-235.

CARmo, D. A. \& SAnguinetTi, Y. I. 1999. Taxonomy and paleocenographical significance of the Krithe genus in the Brazilian continental margin. J. Micropaleont., London, 18(2):111-123.

Chaves, H. A. F. 1983. Introdução geral. In: Projeto Remac. Processos e métodos. Relatório final. Rio de Janeiro, PETROBRÁS/CENPES/SINTEP. v.6, p.7-31.

Coimbra, J. C. \& Ornellas, L. P. 1989. Distribution and ecology of sub-recent Orionininae (Ostracoda) in the Brazilian continental shelf. Revta bras. Geoci., Porto Alegre, 19(2):177-186.

Coimbra, J. C.; Sanguinetti, Y. T. \& Bittencourt-Calcagno, V. M. 1995. Taxonomy and distribution patterns of recent species of Callistocythere Ruggieri, 1953 (Ostracoda) from the Brazilian continental shelf. Revta Esp. Micropaleont., Madrid, 27(3):117-136.

Fauth, G. \& Coimbra, J. C. 1998. Zoogeography of the ostracode genera Auradilus and Radimella (Ostracoda) along the Brazilian continental shelf. N. Jb. Geol. Paläont. Mh., Tübingen, 8(8):463-474.

Forti-Esteves, I. R. 1984. Recent bivalves (Palaeotaxodonta and Pteriomorphia) from de Brazilian continental shelf. Pesquisas, Porto Alegre, 16:190-226.

Francisconi, O.; Costa, M. P. A. et al. 1974. Geologia costeira e sedimentos da plataforma continental brasileira. In: Congresso Brasileiro de Geologia, 28 $8^{\circ}$, Porto Alegre. Anais... Porto Alegre, SBG. v.3, p.322-325.

Hazel, J. E. 1967. Corrections: classification and distribution of the recent Hemicytheridae and Trachyleberididae (Ostracoda) off northeastern North America. J. Paleont., Tulsa, 41:1284-1285.

KemPF, M. 1970. Notes on the benthic bionomy of the N-NE Brazilian shelf. Mar. Biol., Marseille, 5(3):213-224.

Kowsmann, R. O. \& CostA, M. P. A. 1979. Sedimentação quaternária da margem continental brasileira e das águas oceânicas adjacentes. In: ProJeto Remac, Reconhecimento global da margem continental brasileira. Rio de Janeiro, PETROBRÁS/CENPES/SINTEP. v.8, p.1-55.

Martins, L. R. 1984. Aspectos da oceanografia física do Atlântico Sul. Pesquisas, Porto Alegre, 16:76-90.

Mckenzie, K. G. 1967. Recent Ostracoda from Port Phillips Bay, Victoria. Proc. R. Soc. Vic., Victoria, 80(1):61106.

Moore, R. C. \& Pitrat, C. W. 1961. Treatise on Invertebrate Paleontology. Part Q - Arthropoda 3: Crustacea (Ostracoda). New York, Geological Society of America. 442p.

Morkhoven, F. P. C. M. van. 1963. Post-palaeozoic Ostracoda; their morphology, taxonomy and economic use. (generic descriptions). Amsterdan, Elsevier. v.2, 478p.

Pinto, I. D.; Ornellas, L. P. et al. 1978. Recent ostracodes along 7,408km of the Brazilian coast $\left(33^{\circ} 45^{\prime} \mathrm{S}\right.$ to $\left.4^{\circ} 25^{\prime}\right)$. Pesquisas, Porto Alegre, 9:109-120.

Puri, H. S. 1953. The ostracods genus Trachyleberis and its ally Actinocythereis. Amer. Midl. Nat., Notre Dame, 49(1): 171-87.

Ramos, M. I. F. 1996. Taxonomy and zoogeography of the ostracod genera Nanocoquimba and Cornucoquimba 
Ohmert, 1968 from recent sediments on the Brazilian continental shelf. Revta Esp. Micropaleont., Madrid, 28(1):105-128.

Stevenson, M. R.; Dias-Brito, D. et al. 1998. How do cold water biota arrive in a tropical bay near Rio de Janeiro, Brazil? Cont. Shelf Res., Philadelphia, 18:1595-1612.

Recebido em 25.09.2001; aceito em 18.04.2002. 